\title{
A Geometrical Setting for Constructing Nonlinear Lorentz Transformations of the Albano-Dresden Type
}

\author{
Alexandre Lyra ${ }^{1}$ and Marcelo Carvalho ${ }^{2}$ \\ ${ }^{1}$ Observatório do Valongo, Universidade Federal do Rio de Janeiro, 20080-090 Rio de Janeiro, RJ, Brazil \\ ${ }^{2}$ Departamento de Matemática, Universidade Federal de Santa Catarina, 88040-900 Florianópolis, SC, Brazil
}

Correspondence should be addressed to Marcelo Carvalho, marcelo_carv@hotmail.com

Received 30 September 2010; Accepted 3 January 2011

Academic Editor: Myung Kim

Copyright ( 2011 A. Lyra and M. Carvalho. This is an open access article distributed under the Creative Commons Attribution License, which permits unrestricted use, distribution, and reproduction in any medium, provided the original work is properly cited.

\begin{abstract}
We investigate a nonlinear realization of the Lorentz transformation defined in 4-dimensional spacetime, and the corresponding invariant interval. This new interval is not a quadratic form in the standard Minkowski spacetime, then we search for a description where it becomes a quadratic form in an infinite dimensional vector space. In this extended space, we show how to generate two classes of nonlinear transformations, which form a group and interpret the non-linear transformation as compatible relations between atlas of a Lyra manifold.
\end{abstract}

\section{Introduction}

Spacetime structure is characterized at the classical level by the four-dimensional interval $I=\eta_{\mu \nu} x^{\mu} x^{\nu}$ (or $d I=$ $\eta_{\mu \nu} d x^{\mu} d x^{\nu}$ when we consider spacetime modelled as a manifold instead of a vector space) and the corresponding Lorentz transformation $x^{\mu} \rightarrow x^{\prime \mu}=\Lambda_{\nu}^{\mu} x^{\nu}$. Since no experiment can probe spacetime at very small distances, it is possible to consider that at these scales spacetime is described by a modified interval $I_{q}$, and the Lorentz invariance is broken in the sense of not leaving $I_{q}$ invariant. This conjecture was considered by some authors in several different contexts (see [1-3] and references therein).

In our work, we investigate a model proposed originally by Albano and Dresden [4-7] where the standard interval $I$ and the Lorentz transformation are replaced by

$$
\begin{gathered}
I_{q}=\eta_{\mu \nu} x^{\mu} x^{\nu}+\hat{f}, \\
x^{\prime \mu}=h_{\Lambda} \Lambda_{\nu}^{\mu} x^{\nu} .
\end{gathered}
$$

Here, $\hat{f}$ is an arbitrary function depending on the ratio $x^{\mu} / x^{0}$, and $h_{\Lambda}$ is a function whose form is determined by imposing the nonlinear transformation leaves $I_{q}$ invariant.
The purpose of our work is twofold. First, to provide a general mathematical construction for spacetime models that are based on (1) and (2) and that include, as a particular case, the original Albano-Dresden model. Second, to clarify and revisit some aspects of the Albano-Dresden model, specially those concerning the interpretation of the nonlinear transformation. Like Albano and Dresden, we present no extensive analysis on the possible quantum effects that could justify a change in the spacetime interval, for example, $I \rightarrow$ $I_{q}$, as proposed in (1).

Our mathematical construction is based on the attempt to interpret $I_{q}$ as a quadratic form in a certain space. Due to the arbitrariness of $f$, it is evident that $I_{q}$ is not a quadratic form in ordinary spacetime. The solution is to consider a family of vector spaces $\left\{E_{x}\right\}_{x \in M}$ and to introduce sections $s \in \prod_{x \in M} E_{x}$, that is, $s: U \subseteq M \rightarrow \cup_{x \in M} E_{x}$, in terms of which we define an extended space $M \oplus \prod_{x \in M} E_{x}$ where $I_{q}$ is a quadratic form. Then, assuming the nonlinear transformation $x^{\prime}=h_{\Lambda} \Lambda x$ and suitable transformations for the sections we deduce, upon imposing invariance of $I_{q}$, two forms for $h_{\Lambda}$, one determining the original AlbanoDresden transformation and the other a new transformation not contemplated in their model. 
The interpretation of the nonlinear transformation needs a careful analysis. Albano and Dresden show in $[6,7]$ that by introducing new variables $\xi, \xi^{\prime}$ as $\xi^{\mu}:=$ $x^{\mu} \sqrt{1+\left(1 / x^{2}\right) f\left(x^{i} / x^{0}\right)}, \xi^{\prime \mu}:=x^{\prime \mu} \sqrt{1+\left(1 / x^{\prime 2}\right) f\left(x^{\prime i} / x^{\prime 0}\right)}$ the nonlinear transformation becomes linear, that is, $\xi \rightarrow$ $\xi^{\prime}=\Lambda \xi$. Then, one can associate the violation of Lorentz symmetry to the failure on defining the variables $\xi$, $\xi^{\prime}$. This occurs for points satisfying $1+\left(1 / x^{2}\right) f\left(x^{i} / x^{0}\right)<0$ that defines a distinguished region of spacetime. However, theoretical and observational aspects indicate there is no preferred region like this. Therefore, it is necessary to clarify the meaning of the nonlinear transformation in a different way. We achieve this by treating the nonlinear transformation as a passive transformation between charts of two compatible atlas that are employed by two different observers. The possibility to linearize the nonlinear transformation, that is, to reduce it to the standard Lorentz form, is seen as an active transformation between charts of two compatible atlas used by the same observer. With this view, we are able to avoid inferences on the existence of specific spacetime regions where Lorentz violation occurs. Instead of that, we show that the violation can be considered as an effect related to specific choices of atlas and, therefore, it concerns the observer in its relation to the spacetime rather than being an intrinsic property of spacetime.

Our work is organized as follows. In Section 2 we build an infinite dimensional vector space where we define $I_{q}$ and derive the nonlinear transformations as transformations that leave $I_{q}$ invariant. We also show that they obey a group structure. In Section 3 we assume spacetime is endowed with a Lyra structure and interpret the linear and the nonlinear transformations in terms of compatible relations between atlas used by two observers. Finally, in Section 4 we give a heuristic interpretation to the function $\hat{f}$ showing how it arises as a consequence of the uncertainty principle in the measurement of position. We also indicate how the shift from linear to nonlinear Lorentz transformation may form a theoretical framework for the fluctuation of the spacetime metric.

Henceforth, we refer the interval $I_{q}$ as the Albano-Dresden form. The nonlinear transformation (2) is called AlbanoDresden transformation despite the form of $h_{\Lambda}$ we consider.

\section{The Nonlinear Transformation and the Spacetime Structure}

2.1. Defining the Extended Space and the Albano-Dresden Form $I_{q}$. Let us assume spacetime is a pair $(M, g)$ with $M$ a 4 -dimensional real vector space and $g$ a bilinear form that is symmetric, nondegenerate, and of rank 2 . Let $\left\{e_{0}, e_{1}, e_{2}, e_{3}\right\}$ be an orthonormal basis for $M$ in terms of which we write $g\left(e_{\mu}, e_{\nu}\right)=\eta_{\mu \nu}$, with $\eta_{\mu \nu}=(-,+,+,+)$ or $(+,-,-,-)$. The associated quadratic form is written as $I(x)=\eta_{\mu \nu} x^{\mu} x^{\nu}$ where $x \equiv x_{(B A)}:=x_{B}-x_{A}$ will always refer to the difference between the coordinates of two events $x_{A}=\left(x_{A}^{\mu}\right)$ and $x_{B}=$ $\left(x_{B}^{\mu}\right)$. If we consider an event $x_{A}=\left(x_{A}^{\mu}\right)$ and write $I(x)=$ $\eta_{\mu \nu} x^{\mu} x^{\nu}$ we understand that $x \equiv x_{(A O)}:=x_{A}-x_{O}$ with $x_{O}=(0,0,0,0)$.
Now, let $\Omega \equiv\left\{E_{x}\right\}_{x \in M}$ be a family of real vector spaces indexed by spacetime $M$. We assume $E_{x}$ is 4-dimensional with basis $\left\{\theta_{\mu x}\right\}$. The direct product space $\prod_{x \in M} E_{x}$ consists of maps $s: M \rightarrow \cup_{x \in M} E_{x}$ where $s(x) \in E_{x}$. Following the notation of [8], we write $s=\left(s_{x}\right)_{x \in M}$, with $s_{x}:=s(x)=$ $s_{x}^{\mu} \theta_{\mu x} \in E_{x}\left(s_{x}^{\mu} \in R\right)$, and define the sum of elements $s, s^{\prime} \in$ $\prod_{x \in M} E_{x}$ as

$$
s+s^{\prime} \equiv\left(s_{x}\right)_{x \in M}+\left(s_{x}^{\prime}\right)_{x \in M}:=\left(s_{x}+s_{x}^{\prime}\right)_{x \in M}
$$

while the product of $s \in \prod_{x \in M} E_{x}$ by a scalar $\lambda \in R$ is defined as

$$
\lambda s \equiv \lambda\left(s_{x}\right)_{x \in M}:=\left(\lambda s_{x}\right)_{x \in M} .
$$

In this way, $\prod_{x \in M} E_{x}$ becomes a vector space. Let us consider the elements $\theta_{\mu[y]}:=\left(\delta_{x y} \theta_{\mu x}\right)_{x \in M}$ which allows us to write $s=\sum_{y \in M} s_{y}^{\mu} \theta_{\mu[y]}$. In fact, from the sum and product by scalar defined in $\prod_{x \in M} E_{x}$ we have

$$
\begin{aligned}
\sum_{y \in M} s_{y}^{\mu} \theta_{\mu[y]} & =\sum_{y \in M}\left(s_{y}^{\mu} \delta_{x y} \theta_{\mu x}\right)_{x \in M}=\left(\left(\sum_{y \in M} s_{y}^{\mu} \delta_{x y}\right) \theta_{\mu x}\right)_{x \in M} \\
& =\left(s_{x}^{\mu} \theta_{\mu x}\right)_{x \in M}=s .
\end{aligned}
$$

We notice that $s=\sum_{y \in M} s_{y}^{\mu} \theta_{\mu[y]}$ is understood as a formal expression that attains a rigorous meaning as a finite sum if we consider $s$ having finite support and, in this case, the exterior product becomes the external direct sum $\oplus_{x \in M} E_{x}$. (In the general case, the meaning given to $s=\sum_{y \in M} s_{y}^{\mu} \theta_{\mu[y]}$ must be understood as similar to the same formal meaning one gives to the expression representing a divergent series of real numbers, or to the path integral measure $\mathscr{D} \phi$.)

Consider now the exterior direct sum of $M$ and $\prod_{x \in M} E_{x}$, that is, $M \oplus\left(\prod_{x \in M} E_{x}\right) \ni(x, s)$. Henceforth, we denote $(x, s)$ as $x \oplus s$. Let us introduce elements $\tilde{e}_{\mu}:=\left(e_{\mu}, 0\right), \widetilde{\theta}_{\mu y}:=$ $\left(0, \theta_{\mu[y]}\right)$, in terms of each we write $x \oplus s=x^{\mu} \widetilde{e}_{\mu}+\sum_{y \in M} s_{y}^{\mu} \widetilde{\theta}_{\mu y}$. Any finite set of the form $\left\{\tilde{\theta}_{\mu_{1} x_{1}}, \ldots, \tilde{\theta}_{\mu_{r} x_{r}}\right\} \subset \prod_{x \in M} E_{x}$ is linearly independent (with $x_{i} \neq x_{j}$ ), and we define a bilinear form in $M \oplus \prod_{x \in M} E_{x}$ as follows:

$$
\begin{gathered}
Q\left(\tilde{e}_{\mu}, \tilde{e}_{\nu}\right)=\eta_{\mu \nu}, \quad Q\left(\tilde{e}_{\mu}, \tilde{\theta}_{\mu x}\right)=0, \\
Q\left(\tilde{\theta}_{\mu x}, \tilde{\theta}_{\nu x}\right)=\eta_{\mu \nu} f^{2}(x), \quad Q\left(\tilde{\theta}_{\mu x}, \tilde{\theta}_{\nu y}\right)=0 \quad(\text { if } x \neq y),
\end{gathered}
$$

with $f$ an arbitrary nonnull real-valued function of spacetime. (The fact that in (6) we write $f^{2}$ instead of $f$ will become clear in Section 3.1). By linearity, we extend the bilinear form $Q$ to the whole space $M \oplus \prod_{x \in M} E_{x}$ as follows:

$$
\begin{aligned}
Q(x \oplus s, y \oplus q) & =Q(x, y)+Q(s, q) \\
& =\eta_{\mu \nu} x^{\mu} y^{\nu}+\sum_{z, w} \delta_{z w} f^{2}(z) \eta_{\mu \nu} s_{z}^{\mu} q_{w}^{\nu} \\
& =\eta_{\mu \nu} x^{\mu} y^{\nu}+\sum_{z} f^{2}(z) \eta_{\mu \nu} s^{\mu}(z) q^{\nu}(z) .
\end{aligned}
$$


Define now a map

$$
\begin{gathered}
\Delta_{x}: \prod_{x \in M} E_{x} \longrightarrow \prod_{x \in M} E_{x}, \\
s \longrightarrow \Delta_{x} s:=s_{x}^{\mu} \theta_{\mu[x]}
\end{gathered}
$$

and the subset

$M \oplus_{\Delta} \prod_{x \in M} E_{x}:=\left\{\left(x, \Delta_{x} s\right) \in M \oplus \prod_{x \in M} E_{x}: x \in M, s \in \prod_{x \in M} E_{x}\right\}$.

Restricted to these elements, the bilinear form assumes the value

$$
\begin{aligned}
Q\left(x \oplus \Delta_{x} s, y \oplus \Delta_{y} q\right) & =Q(x, y)+Q\left(\Delta_{x} s, \Delta_{y} q\right) \\
& =\eta_{\mu \nu} x^{\mu} y^{\nu}+\delta_{x y} f^{2}(x) \eta_{\mu \nu} s^{\mu}(x) q^{\nu}(y)
\end{aligned}
$$

In particular, we obtain $I_{q}$ as

$$
\begin{aligned}
I_{q}(x, s) & :=Q\left(x \oplus \Delta_{x} s, x \oplus \Delta_{x} s\right) \\
& =\eta_{\mu \nu} x^{\mu} x^{\nu}+f^{2}(x) \eta_{\mu \nu} s^{\mu}(x) s^{\nu}(x)
\end{aligned}
$$

which leads us to identify the function $\hat{f}$ of the original Albano-Dresden transformation $[4,5]$ as

$$
\widehat{f}(x) \equiv f^{2}(x) \eta_{\mu \nu} s^{\mu}(x) s^{\nu}(x) .
$$

We identify $M$ with $\left.M \oplus_{\Delta} \prod_{x \in M} E_{x}\right|_{s=0}$ by the correspondence $\left.M \ni x \leftrightarrow(x, 0) \in M \oplus_{\Delta} \prod_{x \in M} E_{x}\right|_{s=0}$. Then, $I=x^{2}$ becomes a particular case of $I_{q}=x^{2}+s^{2} f^{2}$ when we take $s=0$.

2.2. Deriving Transformations that Leave $I_{q}$ Invariant. The analysis of the invariance of the interval $I_{q}=x^{2}+\hat{f}$ must take into account transformations of both $x$ and $s$, that is, $(x, s(x)) \rightarrow\left(x^{\prime}, s^{\prime}\left(x^{\prime}\right)\right)$. In what follows, we analyze two types of transformations for the sections $s$ that will determine two classes of nonlinear transformations.

2.2.1. Type I Transformations. Let us assume transformations $(x, s(x)) \rightarrow\left(x^{\prime}, s^{\prime}\left(x^{\prime}\right)\right)$ having the form

$$
\begin{aligned}
& x^{\prime \mu}:=h_{\Lambda}(x) \Lambda_{\nu}^{\mu} x^{\nu}, \\
& s^{\prime \mu}\left(x^{\prime}\right):=\Lambda_{\nu}^{\mu} s^{\nu}(x) .
\end{aligned}
$$

Imposing invariance of $I_{q}$ on $M \oplus_{\Delta} \prod_{x \in M} E_{x}$

$$
\begin{gathered}
Q\left(x^{\prime} \oplus \Delta_{x^{\prime}} s^{\prime}, x^{\prime} \oplus \Delta_{x^{\prime}} s^{\prime}\right)=Q\left(x \oplus \Delta_{x} s, x \oplus \Delta_{x} s\right), \\
\therefore \quad x^{2} h_{\Lambda}^{2}(x)+s^{2}(x) f^{\prime 2}\left(x^{\prime}\right)=x^{2}+f^{2}(x) s^{2}(x), \\
h_{\Lambda}^{2}(x)=1+\frac{s^{2}(x)}{x^{2}}\left(f^{2}(x)-f^{\prime 2}\left(x^{\prime}\right)\right) .
\end{gathered}
$$

In order to be able to determine $h_{\Lambda}$ from this last equation, it is sufficient to impose that $f(x)$ depends on the ratio $x^{\mu} / x^{0}$, that is, $f \equiv f\left(x^{\mu} / x^{0}\right)$, which gives

$$
h_{\Lambda}(x, s(x))=\sqrt{1+\frac{s^{2}(x)}{x^{2}}\left(f^{2}(\hat{x})-f^{\prime 2}(\Lambda \hat{x})\right)}
$$

with type I transformations assuming the form

$$
\begin{gathered}
x^{\prime \mu}=\Lambda_{\nu}^{\mu} x^{\nu} \sqrt{1+\frac{s^{2}(x)}{x^{2}}\left(f^{2}(\hat{x})-f^{\prime 2}(\Lambda \hat{x})\right)}, \\
s^{\prime \mu}\left(x^{\prime}\right)=\Lambda_{\nu}^{\mu} s^{\nu}(x),
\end{gathered}
$$

where we have used the notation

$$
\hat{x}^{\mu}:=\frac{x^{\mu}}{x^{0}}, \quad(\Lambda \hat{x})^{\mu}:=\frac{\Lambda_{\nu}^{\mu} x^{\nu}}{\Lambda_{\alpha}^{0} x^{\alpha}} .
$$

In order to exhibit the group structure of type I transformations we consider two consecutive transformations

$$
\begin{aligned}
&(x, s(x)) \stackrel{L}{\stackrel{L\left(\Lambda_{(1)}\right)}{\rightarrow}}\left(x^{\prime}, s^{\prime}\left(x^{\prime}\right)\right) \stackrel{L\left(\Lambda_{(2)}\right)}{\rightarrow}\left(x^{\prime}, s^{\prime \prime}\left(x^{\prime \prime}\right)\right): \\
& x^{\prime}=h_{\Lambda_{(1)}}(x) \Lambda_{(1)} x \\
&=\sqrt{1+\frac{s^{2}(x)}{x^{2}}\left(f^{2}(\hat{x})-f^{\prime 2}\left(\Lambda_{(1)} \hat{x}\right)\right)} \Lambda_{(1)} x, \\
& s^{\prime}\left(x^{\prime}\right)=\Lambda_{(1)} s(x), \\
& x^{\prime \prime}=h_{\Lambda_{(2)}}\left(x^{\prime}\right) \Lambda_{(2)} x^{\prime} \\
&=\sqrt{1+\frac{s^{\prime 2}\left(x^{\prime}\right)}{x^{\prime 2}}\left(f^{\prime 2}\left(\hat{x}^{\prime}\right)-f^{\prime \prime 2}\left(\Lambda_{(2)} \hat{x}^{\prime}\right)\right) \Lambda_{(2)} x^{\prime},} \\
& s^{\prime \prime}\left(x^{\prime \prime}\right)=\Lambda_{(2)} s^{\prime}\left(x^{\prime}\right) .
\end{aligned}
$$

Using that

$$
\begin{aligned}
& \sqrt{1+\frac{s^{\prime 2}\left(x^{\prime}\right)}{x^{\prime 2}}\left(f^{\prime 2}\left(\hat{x}^{\prime}\right)-f^{\prime \prime 2}\left(\Lambda_{(2)} \hat{x}^{\prime}\right)\right)} \\
& =\frac{1}{h_{\Lambda_{(1)}(x)}} \sqrt{1+\frac{s^{2}(x)}{x^{2}}\left(f^{2}(\hat{x})-f^{\prime \prime 2}\left(\Lambda_{(2)} \Lambda_{(1)} \hat{x}\right)\right)}
\end{aligned}
$$

we obtain $x^{\prime \prime}=h_{\Lambda_{(2)} \Lambda_{(1)}}(x) \Lambda_{(2)} \Lambda_{(1)} x$, while for the sections it is immediate that $s^{\prime \prime}\left(x^{\prime \prime}\right)=\Lambda_{(2)} \Lambda_{(1)} s(x)$. Thus, we conclude that $L\left(\Lambda_{(2)}\right) \circ L\left(\Lambda_{(1)}\right)=L\left(\Lambda_{(2)} \Lambda_{(1)}\right)$. In particular, the inverse of transformation (16) is written as

$$
\begin{gathered}
x=h_{\Lambda^{-1}}\left(x^{\prime}\right) \Lambda^{-1} x^{\prime}=\sqrt{1+\frac{s^{\prime 2}\left(x^{\prime}\right)}{x^{\prime 2}}\left(f^{\prime 2}\left(\hat{x}^{\prime}\right)-f^{2}\left(\Lambda^{-1} \hat{x}^{\prime}\right)\right)} \\
s(x)=\Lambda^{-1} s^{\prime}\left(x^{\prime}\right)
\end{gathered}
$$

and one verifies that $h_{\Lambda^{-1}}\left(x^{\prime}\right)=1 / h_{\Lambda}(x)$. We have then showed the group structure of type I transformations. 
2.2.2. Type II Transformations. These transformations are defined by

$$
\begin{gathered}
x^{\prime \mu}:=h_{\Lambda}(x) \Lambda_{\nu}^{\mu} x^{\nu}, \\
s^{\prime \mu}\left(x^{\prime}\right):=h_{\Lambda}(x) \Lambda_{\nu}^{\mu} s^{\nu}(x) .
\end{gathered}
$$

Now, imposing invariance of $I_{q}$ we obtain $h_{\Lambda}^{2}(x)\left(x^{2}+\right.$ $\left.s^{2}(x) f^{\prime 2}\left(x^{\prime}\right)\right)=x^{2}+s^{2}(x) f^{2}(x)$, and in order to determine $h_{\Lambda}$ we again consider sections whose argument is $\hat{x}^{\mu}:=x^{\mu} / x^{0}$, with $(\Lambda \hat{x})^{\mu}:=\Lambda_{\nu}^{\mu} x^{\nu} / \Lambda_{\alpha}^{0} x^{\alpha}$. We obtain

$$
h_{\Lambda}(x, s(x))=\sqrt{\frac{x^{2}+s^{2}(x) f^{2}(\hat{x})}{x^{2}+s^{2}(x) f^{\prime 2}(\Lambda \hat{x})}}
$$

and type II transformations assume the form

$$
\begin{gathered}
x^{\prime \mu}:=\Lambda_{\nu}^{\mu} x^{\nu} \sqrt{\frac{x^{2}+s^{2}(x) f^{2}(\hat{x})}{x^{2}+s^{2}(x) f^{\prime 2}(\Lambda \hat{x})}}, \\
s^{\prime \mu}\left(x^{\prime}\right):=\Lambda_{\nu}^{\mu} s^{\nu}(x) \sqrt{\frac{x^{2}+s^{2}(x) f^{2}(\hat{x})}{x^{2}+s^{2}(x) f^{\prime 2}(\Lambda \hat{x})}} .
\end{gathered}
$$

From these expressions it is straightforward to check the group structure of type II transformations.

Remarks. (1) For both type I and II transformations we notice that $f$ depends on the ratio $x^{\mu} / x^{0}$, which has no dimension. From the expression of $I_{q}=x^{2}+s^{2} f^{2}(\hat{x})$ it is then necessary to introduce a unit of length in $s$ which implies the existence of a fundamental length in the model $[4,5]$.

(2) $s(x) \in E_{x}, s^{\prime}\left(x^{\prime}\right) \in E_{x^{\prime}}$ are vectors belonging to different vector spaces, and each one is characterized numerically by the components $s^{\mu}(x), s^{\prime \mu}\left(x^{\prime}\right) \in R$. Therefore, transformation $s^{\mu}(x) \rightarrow s^{\prime \mu}\left(x^{\prime}\right)$ given in (16) and (23) is seen as a transformation in the space of real maps defined on $M$.

(3) For a fixed $f$ and its transformed function $f^{\prime}\left(x^{\prime}\right)$, the nonlinear transformations of type I and II are defined only in a restricted spacetime region $[6,7]$. Therefore, the only way to have nonlinear transformations defined through all spacetime is to employ several functions $f_{a}: U_{a} \subset M \rightarrow R$ such that in the domains $V_{a} \subseteq U_{a}$ where $h_{\Lambda}$ is defined we have $\cup V_{a}=M$.

(4) If we consider $s \in \prod_{x \in M} E_{x}$ satisfying $s^{2}(x)=1$, type I transformations become

$$
x^{\prime \mu}=\Lambda_{\nu}^{\mu} x^{\nu} \sqrt{1+\frac{1}{x^{2}}\left(f^{2}(\hat{x})-f^{\prime 2}(\Lambda \hat{x})\right)}
$$

that reduce to the standard form proposed originally by Albano and Dresden [4-7]. This choice, however, is not compatible with type II transformations as it fixes $h_{\Lambda}=1$.

\section{Interpreting the Nonlinear Transformation}

Here, we identity $E_{x}=T_{x} M$, and spacetime becomes a particular case of $M \oplus_{\Delta} \Gamma(T M)$ when we take $s=0$, that is, $\left.M \sim M \oplus_{\Delta} \Gamma(T M)\right|_{s=0}$. In order to interpret the nonlinear transformations we assume spacetime is a Lyra manifold, and each observer describes spacetime by choosing one appropriate Lyra structure, perhaps among many possible ones.

3.1. Redefining Spacetime in Terms of a Lyra Manifold. Following [9], we define a Lyra manifold as a set $M$ together with $\mathcal{A}:=\left\{\left(U_{a}, \varphi_{a}, f_{a} ; x_{a}\right)\right\}_{a \in \Omega}$ such that

(i) $\left\{\left(U_{a}, \varphi_{a} ; x_{a}\right)\right\}_{a \in \Omega}$ is a $C^{\infty}$ atlas in $M$,

(ii) $f_{a}: U_{a} \rightarrow R-\{0\}$ is a $C^{\infty}$ map ( $f_{a}$ is called the gauge function on $U_{a}$ ),

(iii) $\mathcal{A}$ is maximal.

Since a maximal atlas is an equivalence class of compatible atlas, it is enough to define a Lyra manifold in terms of an atlas satisfying conditions (i) and (ii). In this sense we say the atlas $\mathcal{A}$ defines a Lyra structure for $M$.

Given $p \in M$, let $\left(U_{a}, \varphi_{a}, f_{a} ; x_{a}\right) \in \mathcal{A}$ with $p \in U_{a}$. Locally, we have $\varphi_{a}(p)=x_{a} \equiv\left(x_{a}^{\mu}\right) \in R^{4}$ and we define a basis for $T_{p} M$ as $\left\{\partial / \partial x_{a}^{\mu}\right\}$. Assume now there is defined a metric on $M$ according to

$$
g\left(\frac{\partial}{\partial x_{a}^{\mu}}, \frac{\partial}{\partial x_{a}^{\nu}}\right)={ }_{a} g_{\mu \nu}\left(x_{a}\right) .
$$

Introduce a new basis for $T_{p} M$ as $\left\{a \theta_{\mu p}\right\}$, with

$$
{ }_{a} \theta_{\mu p}:=f_{a}\left(x_{a}\right) \frac{\partial}{\partial x_{a}^{\mu}},
$$

where $f_{a}\left(x_{a}\right)$ denotes $f_{a} \circ \varphi_{a}^{-1}$. Then

$$
g\left({ }_{a} \theta_{\mu p},{ }_{a} \theta_{\nu p}\right)=f_{a}^{2}\left(x_{a}\right)_{a} g_{\mu \nu}\left(x_{a}\right) .
$$

In this expression, $g_{\mu \nu}$ are the components of the metric relative to the original basis $\left\{\partial / \partial x_{a}^{\mu}\right\}$, therefore it transforms as

$$
{ }_{a_{1}} g_{\mu \nu}\left(x_{a_{1}}\right)=\frac{\partial x_{a_{2}}^{\alpha}}{\partial x_{a_{1}}^{\mu}} \frac{\partial x_{a_{2}}^{\beta}}{\partial x_{a_{1}}^{\nu}} a_{2} g_{\alpha \beta}\left(x_{a_{2}}\right) .
$$

From (26) and (28) we obtain that for all $p \in U_{a_{1}} \cap U_{a_{2}}$

$$
\begin{aligned}
g\left(a_{1} \theta_{\mu p}, a_{1} \theta_{\nu p}\right) & =f_{a_{1}}^{2}\left(x_{a_{1}}\right){ }_{a_{1}} g_{\mu \nu}\left(x_{a_{1}}\right) \Longleftrightarrow g\left(a_{a_{2}} \theta_{\mu p}, a_{2} \theta_{\nu p}\right) \\
& =f_{a_{2}}^{2}\left(x_{a_{2}}\right)_{a_{2}} g_{\mu \nu}\left(x_{a_{2}}\right)
\end{aligned}
$$

which shows that (28) is compatible throughout the charts. One should also observe that relation $Q\left(\tilde{\theta}_{\mu x}, \tilde{\theta}_{\nu x}\right)=\eta_{\mu v} f^{2}(x)$ is a consequence of (27) when we take $E_{p} \equiv T_{p} M$ and consider $g_{\mu \nu}=\eta_{\mu \nu}$. 


\subsection{The Transformations}

Definition 1 (nonlinear Lorentz transformation). Consider extended spacetime $M \oplus_{\Delta} \Gamma(T M)$, with $M$ endowed with Lyra structures $\mathcal{A}_{\mathcal{O}}:=\left\{\left(U_{a}, \varphi_{a}, f_{a} ; x_{a}\right)\right\}, \mathcal{A}_{\mathcal{O}^{\prime}}:=\left\{\left(U_{b}^{\prime}, \varphi_{b}^{\prime}, f_{b}^{\prime} ; x_{b}^{\prime}\right)\right\}$ relative to observers $\mathcal{O}$ and $\mathcal{O}^{\prime}$. Let the spacetime metric (introduced in (27)) be $g_{\mu \nu}^{\prime}=g_{\mu \nu}=\eta_{\mu \nu}$. For every $p \in$ $U_{a} \cap U_{b}^{\prime}$ one defines nonlinear Lorentz transformation as the compatible relations between the charts that have the form

$$
\begin{aligned}
x_{b}^{\prime \mu} & =h_{\Lambda}\left(x_{a}\right) \Lambda_{\nu}^{\mu} x_{a}^{\nu} \\
& =\sqrt{1+\frac{s_{a}^{2}\left(x_{a}\right)}{x_{a}^{2}}\left(f_{a}^{2}\left(\hat{x}_{a}\right)-f_{b}^{\prime 2}\left(\Lambda \hat{x}_{a}\right)\right)} \Lambda_{\nu}^{\mu} x_{a}^{\nu}, \\
x_{b}^{\prime \mu} & =h_{\Lambda}\left(x_{a}\right) \Lambda_{\nu}^{\mu} x_{a}^{\nu} \\
& =\sqrt{\frac{x_{a}^{2}+s_{a}^{2}\left(x_{a}\right) f_{a}^{2}\left(\hat{x}_{a}\right)}{x_{a}^{2}+s_{a}^{2}\left(x_{a}\right) f_{b}^{\prime 2}\left(\Lambda \hat{x}_{a}\right)}} \Lambda_{\nu}^{\mu} x_{a}^{\nu}, \quad \text { (Type II) } .
\end{aligned}
$$

(Type I),

Definition 2 (Lorentz transformation). Consider spacetime $\left.M \oplus_{\Delta} \Gamma(T M)\right|_{s=0}$, with $M$ endowed with single Lyra structures $\mathcal{A}_{\mathcal{O}}:=\{(M, \varphi, f ; x)\}, \mathcal{A}_{\mathcal{O}^{\prime}}:=\left\{\left(M, \varphi^{\prime}, f^{\prime} ; x^{\prime}\right)\right\}$ relative to $\mathcal{O}$ and $\mathcal{O}^{\prime}$. Let the spacetime metric be $g_{\mu \nu}^{\prime}=g_{\mu \nu}=\eta_{\mu \nu}$. For every $p \in M$ one defines Lorentz transformations as the compatible relations between the charts $\mathcal{A}_{\mathcal{O}}, \mathcal{A}_{\mathcal{O}}$, that have the form $x^{\prime}=\Lambda x$.

In the case of Lorentz transformation the gauge functions $f$ and $f^{\prime}$ play no distinguished role, and we could have assumed single differentiable atlas $\mathcal{A}_{\mathcal{O}}:=\{(M, \varphi ; x)\}$, $\mathcal{A}_{\mathcal{O}^{\prime}}:=\left\{\left(M, \varphi^{\prime} ; x^{\prime}\right)\right\}$ instead of Lyra atlas.

3.3. Reducing the Nonlinear Lorentz Transformation to Its Linear Lorentz Form. At this point, the linear and nonlinear Lorentz transformations were set as passive transformations arising as compatible relations between atlas used by two Lorentz-related observers corresponding to single or multichart atlas. A natural question we must analyze is if an observer obtains equivalent descriptions for spacetime whether he uses single or multichart atlas. Thus, we search for compatible atlas $\mathcal{A}_{2}=\left\{\left(U_{a}, \varphi_{a}, f_{a} ; x_{a}\right)\right\} \simeq \mathscr{A}_{1}=$ $\{(M, \varphi, f ; x)\}$ for $\mathcal{O}$, and $\mathcal{A}_{2}^{\prime}=\left\{\left(U_{b}^{\prime}, \varphi_{b}^{\prime}, f_{b}^{\prime} ; x_{b}^{\prime}\right)\right\} \simeq \mathcal{A}_{1}^{\prime}=$ $\left\{\left(M, \varphi^{\prime}, f^{\prime} ; x^{\prime}\right)\right\}$ for $\mathcal{O}^{\prime}$ such that we have (31)

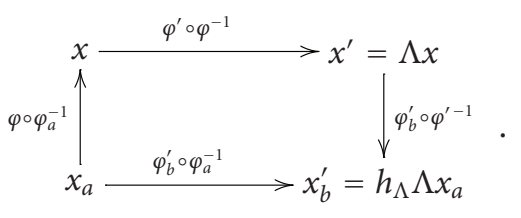

Relative to $\left(U_{a}, \varphi_{a}, f_{a} ; x_{a}\right)$ the invariant interval (11) has the form $I_{a}=x_{a}^{2}+s_{a}^{2}\left(x_{a}\right) f_{a}^{2}\left(\hat{x}_{a}\right)$, while relative to $(M, \varphi ; x)$ its form is $I=x^{2}$. Let us assume that $x_{a}^{\mu}=\Theta(x) x^{\mu}$, and that under this transformation $I_{a}$ reduces to $I$. Then, we obtain $I_{a}=\Theta^{2} x^{2}+s_{a}^{2}\left(x_{a}\right) f_{a}^{2}\left(\hat{x}_{a}\right) \equiv x^{2}$, which imposes $\Theta^{2}(x)=1-$ $\left(s_{a}^{2}\left(x_{a}\right) / x^{2}\right) f_{a}^{2}\left(\hat{x}_{a}\right)$. In order to obtain $\Theta(x)$ it is sufficient to assume that the section depends on $\hat{x}_{a}$, since $\hat{x}_{a}=\hat{x}$ whenever $x_{a}$ is defined. Then $s_{a}\left(\hat{x}_{a}\right)=s_{a}(\hat{x})$ and we obtain $\Theta(x)=$ $\sqrt{1-\left(s_{a}^{2}(\hat{x}) / x^{2}\right) f_{a}^{2}(\hat{x})}$, that is,

$$
\begin{gathered}
x^{\mu} \longrightarrow x_{a}^{\mu}:=x^{\mu} \sqrt{1-\frac{s_{a}^{2}(\hat{x})}{x^{2}} f_{a}^{2}(\hat{x})}, \\
x^{\prime \mu} \longrightarrow x_{b}^{\prime \mu}:=x^{\prime \mu} \sqrt{1-\frac{s_{b}^{\prime 2}\left(\hat{x}^{\prime}\right)}{x^{\prime 2}} f_{b}^{\prime 2}\left(\hat{x}^{\prime}\right)}
\end{gathered}
$$

whose inverse reads as

$$
\begin{gathered}
x_{a}^{\mu} \longrightarrow x^{\mu}:=x_{a}^{\mu} \sqrt{1+\frac{s_{a}^{2}\left(\hat{x}_{a}\right)}{x_{a}^{2}} f_{a}^{2}\left(\hat{x}_{a}\right)}, \\
x_{b}^{\prime \mu} \longrightarrow x^{\prime \mu}:=x_{b}^{\prime \mu} \sqrt{1+\frac{s_{b}^{\prime 2}\left(\hat{x}_{b}^{\prime}\right)}{x_{b}^{\prime 2}} f_{b}^{\prime 2}\left(\hat{x}_{b}^{\prime}\right) .}
\end{gathered}
$$

In order to check if these equations reduce transformations (30) to a linear form we write

$$
\begin{gathered}
x_{b}^{\prime \mu}=\frac{x^{\prime \mu}}{\sqrt{1+\left(s_{b}^{\prime 2}\left(\hat{x}_{b}^{\prime}\right) / x_{b}^{\prime 2}\right) f_{b}^{\prime 2}\left(\hat{x}_{b}^{\prime}\right)}}, \\
x_{a}^{\mu}=\frac{x^{\mu}}{\sqrt{1+\left(s_{a}^{2}\left(\hat{x}_{a}\right) / x_{a}^{2}\right) f_{a}^{2}\left(\hat{x}_{a}\right)}} .
\end{gathered}
$$

Then, for both type I and II transformation we have

$$
\begin{aligned}
x_{b}^{\prime \mu} & =h_{\Lambda}\left(x_{a}\right) \Lambda_{\nu}^{\mu} x_{a}^{\nu} \Longrightarrow x^{\prime \mu} \\
& =\Lambda_{\nu}^{\mu} x^{\nu} h_{\Lambda}\left(x_{a}\right) \sqrt{\frac{x_{b}^{\prime 2}+s_{b}^{\prime 2}\left(\hat{x}_{b}^{\prime}\right) f_{b}^{\prime 2}\left(\hat{x}_{b}^{\prime}\right)}{x_{a}^{2}+s_{a}^{2}\left(\hat{x}_{a}\right) f_{a}^{2}\left(\hat{x}_{a}\right)} \cdot \frac{x_{a}^{2}}{x_{b}^{\prime 2}}} \\
& =\Lambda_{\nu}^{\mu} x^{\nu} \sqrt{\frac{x_{b}^{\prime 2}+s_{b}^{\prime 2}\left(\hat{x}_{b}^{\prime}\right) f_{b}^{\prime 2}\left(\hat{x}_{b}^{\prime}\right)}{x_{a}^{2}+s_{a}^{2}\left(\hat{x}_{a}\right) f_{a}^{2}\left(\hat{x}_{a}\right)}},
\end{aligned}
$$

and since the nonlinear transformation is built in such way that $x_{b}^{\prime 2}+s_{b}^{\prime 2} f_{b}^{\prime 2}=x_{a}^{\prime 2}+s_{a}^{2} f_{a}^{2}$, we end up with $x^{\prime \mu}=\Lambda_{\nu}^{\mu} x^{\nu}$.

In the context of Lyra manifolds, local coordinates are introduced on every $U_{a} \subset M$ provided we have a chart $\left(U_{a}, \varphi_{a}, f_{a} ; x_{a}\right)$. However, depending on the choice of the sections and on the form of the gauge function, the transformation $x=x_{a} \sqrt{1+\left(s_{a}^{2}\left(\hat{x}_{a}\right) / x_{a}^{2}\right) f_{a}^{2}\left(\hat{x}_{a}\right)} \equiv \varphi \circ \varphi_{a}^{-1}\left(x_{a}\right)$ may not be defined in all $\varphi_{a}\left(U_{a}\right) \subset R^{4}$. Besides that, the transformation $x=\varphi \circ \varphi_{a}^{-1}(x)$ is $C^{\infty}$ only if $(1+$ $\left.\left(s_{a}^{2}\left(\hat{x}_{a}\right) / x_{a}^{2}\right) f_{a}^{2}\left(\hat{x}_{a}\right)\right) \neq 0$. Let us assume that this happens in a domain $V_{a} \subseteq U_{a}$. If $\cup_{a} V_{a}=M$, we restrict the original chart $\left(U_{a}, \varphi_{a}, f_{a} ; x_{a}\right)$ to $\left(V_{a},\left.\varphi_{a}\right|_{V_{a}},\left.f_{a}\right|_{V_{a}} ; x_{a}\right)$ obtaining another atlas $\mathcal{A}_{3}:=\left\{\left(V_{a},\left.\varphi_{a}\right|_{V_{a}},\left.f_{a}\right|_{V_{a}} ; x_{a}\right)\right\}$ that is now compatible with $\mathcal{A}_{1}=\{(M, \varphi, f ; x)\}$. Assuming the other observer also defines an atlas $\mathcal{A}_{3}^{\prime}$ compatible with $\mathcal{A}_{1}^{\prime}$, we obtain a complete equivalence between linear and nonlinear Lorentz transformations, the latter reducing to the Lorentz transformation by the use of (33). If $\cup_{a} V_{a} \neq M$, some nonlinear transformation may fail to reduce to the Lorentz transformation. This would 
sign a breaking of Lorentz invariance in the corresponding domain. In our interpretation, this failure is associated with the noncompatibility between differentiable structures, which is quite possible to occur in $R^{4}$. Its origin is related to how observer describes spacetime, that is, it refers to the choice of the differentiable structure, therefore it is not due to an intrinsic property of spacetime. Albano and Dresden suggest the failure on linearizing the transformation is possibly due to quantum mechanical effects. Here, combining their view with our interpretation, we should analyze the extent to which quantum mechanical effects can originate changes in the differentiable structure of spacetime. (For another perpective on this see [10].)

\section{Some Physical Insights}

4.1. A Heuristic Interpretation for $\hat{f}$. The function $\hat{f}$ was interpreted by Dresden as a contribution to the classical interval $I=c^{2} t^{2}-\vec{x}^{2}$ due to quantum mechanics effects $[4,5]$. As an example, they considered a particular situation of two atoms in interaction and showed how the uncertainty in the position of the atoms determines a change in the classical interval. However, it is not clear in their analysis how the function $\hat{f}$ relates, for example, to the wave function of the two atoms. Here, we will show how this arises.

Let us consider two events $A=\left(t_{A}, \vec{x}_{A}\right), B=\left(t_{B}, \vec{x}_{B}\right)$ as measured by an observer $\mathcal{O}$. Event $A$ corresponds to the emission of a quanta by an atom at instant $t_{A}$ and at position $\vec{x}_{A}$. Event $B$ corresponds to the absorption of the quanta by another atom at instant $t_{B}$ and at position $\vec{x}_{B}$. The 4 -interval associated to the events

$$
I\left(t_{B}-t_{A}, \vec{x}_{B}-\vec{x}_{A}\right)=c^{2}\left(t_{A}-t_{B}\right)^{2}-\left|\vec{x}_{A}-\vec{x}_{B}\right|^{2}
$$

is an invariant for any observer that is Lorentz related to $\mathcal{O}$. However, this expression assumes observer $\mathcal{O}$ can determine precisely the positions of both atoms. From a quantum mechanical perspective there is an uncertainty on the position of each atom, therefore we must replace the term $\left|\vec{x}_{A}-\vec{x}_{B}\right|^{2}$ by an average value. Heuristically, let us conceive each atom as oscillating around fixed positions, for example, $\vec{x}_{A}$ and $\vec{x}_{B}$, with $\vec{\xi}_{A}, \vec{\xi}_{B}$ representing small deviations from these values. Then, the effective position of each atom may be written as $\vec{x}_{A}+\vec{\xi}_{A}$ and $\vec{x}_{B}+\vec{\xi}_{B}$. Let us assume the wave function of the system at the instant $t\left(t_{A} \leq t \leq t_{B}\right)$ is described by

$$
\begin{aligned}
\Psi\left(\vec{x}_{A}, \vec{x}_{B} ; \vec{\xi}_{A}, \vec{\xi}_{B}, t\right) \equiv & \Psi_{A}\left(\vec{x}_{B}-\vec{x}_{A} ; \vec{\xi}_{A}, t\right) \\
& \times \Psi_{B}\left(\vec{x}_{B}-\vec{x}_{A} ; \vec{\xi}_{B}, t\right)
\end{aligned}
$$

and normalized such that

$$
\begin{aligned}
& \int d \vec{\xi}_{A}\left|\Psi_{A}\left(\vec{x}_{B}-\vec{x}_{A} ; \vec{\xi}_{A}, t\right)\right|^{2}=1, \\
& \int d \vec{\xi}_{B}\left|\Psi_{B}\left(\vec{x}_{B}-\vec{x}_{A} ; \vec{\xi}_{B}, t\right)\right|^{2}=1
\end{aligned}
$$

with all values of $\xi_{A}^{j}, \xi_{B}^{j}$ being equally probable, that is,

$$
\int d \vec{\xi}_{A} \xi_{A}^{i}\left|\Psi_{A}\right|^{2}=\int d \vec{\xi}_{B} \xi_{B}^{i}\left|\Psi_{B}\right|^{2}=0 .
$$

Since $\vec{x}_{A}, \vec{x}_{B}$ correspond to fixed positions their role on the wave function is just of being parameters, the functional dependence of $\Psi$ being determined by $\vec{\xi}_{A}, \vec{\xi}_{B}$. This justify the normalization of $\Psi$ as integrals on $\vec{\xi}_{A}, \vec{\xi}_{B}$. Besides that, admitting space is homogeneous and isotropic the wave function should depend on $\left|\vec{x}_{B}-\vec{x}_{A}\right|$. Now, in the expression for the interval (36), we replace $\left|\vec{x}_{A}-\vec{x}_{B}\right|^{2}$ by the average value of $\left|\left(\vec{x}_{B}+\vec{\xi}_{B}\right)-\left(\vec{x}_{A}+\vec{\xi}_{A}\right)\right|^{2}$, that is,

$$
\begin{aligned}
& \int d \vec{\xi}_{A} d \vec{\xi}_{B} \delta_{i j}\left(x_{B}^{i}+\xi_{B}^{i}-\left(x_{A}^{i}+\xi_{A}^{i}\right)\right) \\
& \quad \times\left(x_{B}^{j}+\xi_{B}^{j}-\left(x_{A}^{j}+\xi_{A}^{j}\right)\right)\left|\Psi\left(\vec{x}_{A}, \vec{x}_{B} ; \vec{\xi}_{A}, \vec{\xi}_{B}, t\right)\right|^{2}
\end{aligned}
$$

which gives

$$
\begin{aligned}
I_{q}\left(t_{B}-\right. & \left.t_{A}, \vec{x}_{B}-\vec{x}_{A}\right) \\
= & I\left(t_{B}-t_{A}, \vec{x}_{B}-\vec{x}_{A}\right) \\
& \quad-\delta_{i j}\left[\int d \vec{\xi}_{A} \xi_{A}^{i} \xi_{A}^{j}\left|\Psi_{A}\right|^{2}+\int d \vec{\xi}_{B} \xi_{B}^{i} \xi_{B}^{j}\left|\Psi_{B}\right|^{2}\right]
\end{aligned}
$$

with

$$
\hat{f} \equiv-\delta_{i j}\left[\int d \vec{\xi}_{A} \xi_{A}^{i} \xi_{A}^{j}\left|\Psi_{A}\right|^{2}+\int d \vec{\xi}_{B} \xi_{B}^{i} \xi_{B}^{j}\left|\Psi_{B}\right|^{2}\right] .
$$

In this expression, the function $\hat{f}$ is related to the wave function of the system, therefore, it is seen as a quantum effect that is not present in the classical theory. Some criticism could arise if someone inadvertently claims a dependence of $\hat{f}$ on the instant $t$ used to calculate (40). This would result in an interval $I_{q}\left(t_{B}-t_{A}, \vec{x}_{B}-\vec{x}_{A} ; t\right)$ whose dependence on $t$ would be meaningless. In fact, this is not the case as there is no distinguished cause affecting the atoms while the radiation propagates, therefore, it is reasonable to assume that the average (40) does not depend on $t$ or, equivalently, that the wave function is stationary.

\subsection{A Physical Interpretation for $s$ and the Effective Metric for} Spacetime. In our model, the sections are the main elements in the transition from ordinary spacetime $M$ to extended spacetime $M \oplus_{\Delta} \Gamma(T M)$, and the most significant aspect of this transition is the change of the interval from $I=x^{2}$ to $I_{q}=x^{2}+s^{2} f^{2}$. In the previous section, we showed in a heuristic way how $\hat{f}$ could be thought as related to the deviations $\delta \vec{x}=\vec{\xi}$ in the measurement of the position $\vec{x}$ of a physical system. Now, as an intrinsic property of spacetime, we axiomatize that spacetime is described by coordinates and sections $s(x)$, which accounts for the fluctuation of the spacetime coordinates, for example, $x \rightarrow x+s$ when, by any means, we wish to measure it. With this interpretation, 
ordinary spacetime $M$ is obtained when $s=0$ that physically means the interval is $I=x^{2}$ and there is no fluctuation on the spacetime coordinates, in agreement with the classical regime.

We can analyze other aspects brought by the sections, for example, the possibility of interpreting the sections as the source of an effective metric for spacetime. We consider two cases.

4.2.1. Case 1. Let us consider local sections $s: U_{a} \subseteq M \rightarrow$ $T M, \cup_{a} U_{a}=M$, that are analytic in their domain. Then, its Taylor series around a certain $x_{0} \in U_{a}$ is written as

$$
\begin{aligned}
s^{\mu}(x)= & s^{\mu}\left(x_{0}\right)+s_{, \lambda}^{\mu}\left(x_{0}\right)\left(x^{\lambda}-x_{0}^{\lambda}\right) \\
& +\frac{1}{2} s_{, \lambda \tau}^{i}\left(x_{0}\right)\left(x^{\lambda}-x_{0}^{\lambda}\right)\left(x^{\tau}-x_{0}^{\tau}\right)+\cdots
\end{aligned}
$$

with $s_{, \lambda}^{\mu}\left(x_{a}\right)=\left.\left(\partial s^{\mu}(x) / \partial x^{\lambda}\right)\right|_{x_{a}}$. From (11) we obtain after collecting the terms

$$
\begin{aligned}
I_{q}\left(x \oplus \Delta_{x} s\right)= & {\left[\eta_{\lambda \tau}+\eta_{\mu \nu} f^{2}\left(s^{\mu}\left(x_{0}\right) s_{, \lambda \tau}^{\nu}\left(x_{0}\right)+s_{, \lambda}^{\mu}\left(x_{0}\right) s_{, \tau}^{\nu}\left(x_{0}\right)\right)\right] } \\
& \times x^{\lambda} x^{\tau}+h\left(x, x_{0}, s\left(x_{0}\right) \ldots\right),
\end{aligned}
$$

where $h\left(x, x_{0}, s\left(x_{0}\right) \ldots\right)$ includes all non-quadratic contributions on $x$. The quadratic term in $x$ plays the role of an effective metric for spacetime $M$ that we identify as

$$
g_{\lambda \tau}(x)=\eta_{\lambda \tau}+\eta_{\mu \nu} f^{2}\left(s^{\mu}\left(x_{0}\right) s_{, \lambda \tau}^{\nu}\left(x_{0}\right)+s_{, \lambda}^{\mu}\left(x_{0}\right) s_{, \tau}^{\nu}\left(x_{0}\right)\right) .
$$

Another possibility to arrive at this metric is by considering the embedding problem as described in $[11,12]$, that is, we search for a smooth manifold $(N, \hat{g})$ with $\operatorname{dim} N \geq \operatorname{dim} M$ and such that $(M, g)$ is embedded into $N$. Lets us consider $N$ as an 8-dimensional manifold with local coordinates denoted by $\left(Y^{A}\right)=\left(Y^{\alpha}, Y^{\alpha+4}\right), \alpha=1,2,3,4$. Then, the problem is to find $Y^{A}=Y^{A}\left(x^{\alpha}\right)$ such that

$$
g_{\mu \nu}=\hat{g}_{A B} \frac{\partial Y^{A}}{\partial x^{\mu}} \frac{\partial Y^{B}}{\partial x^{\nu}}
$$

Defining $\left(Y^{A}(x)\right):=\left(x^{\alpha}, s^{\alpha}(x)\right)$ with $s=\left(s^{\alpha}\right): U \rightarrow R^{4}$ an embedding, and

$$
\left(\hat{g}_{A B}\right)=\left(\begin{array}{cc}
\eta_{\alpha \beta} & 0 \\
0 & \hat{g}_{\alpha \beta}
\end{array}\right)
$$

with

$$
\widehat{g}_{\lambda \tau}(x):=\frac{1}{2} \eta_{\mu \nu} f^{2} \frac{\partial x^{\alpha}}{\partial s^{\lambda}} \frac{\partial x^{\beta}}{\partial s^{\tau}}\left(\left.\partial_{\alpha} \partial_{\beta}\left(s^{\mu} s^{\nu}\right)\right|_{x=x_{0}}\right)
$$

it is immediate to check that (46) determines (45). Then $(M, g)$ is seen as isometrically embedded into $(N, \hat{g})$. By an appropriate choice of sections and of $\hat{f}$, it is possible to obtain an effective metric that has a nonvanishing curvature tensor.
4.2.2. Case 2. Consider now a curve joining two spacetime points $x_{A}=x\left(\tau_{A}\right), x_{B}=x\left(\tau_{B}\right)$. Let us identify the classical interval as

$$
I=\int_{\tau_{A}}^{\tau_{B}} \eta_{\mu \nu} \frac{d x^{\mu}}{d \tau} \frac{d x^{\nu}}{d \tau} d \tau
$$

The quantum fluctuation $I_{q}$ represents a deviation of $I$ that we identify in two equivalent ways. First, from the interpretation we gave to the sections we consider that at the quantum level there are fluctuations on the coordinates that change the original path joining the points $x_{A}, x_{B}$ to a different path given now by

$$
\tilde{x}^{\mu}:=x^{\mu}+s^{\mu}(x)
$$

with the flat metric $\eta_{\mu \nu}$ being kept fixed, and in terms of which we write

$$
\begin{aligned}
I_{q} & =\int_{\tau_{A}}^{\tau_{B}} \eta_{\mu \nu} \frac{d \tilde{x}^{\mu}}{d \tau} \frac{d \tilde{x}^{\nu}}{d \tau} d \tau \\
& =\int_{\tau_{A}}^{\tau_{B}}\left(\eta_{\mu \nu}+\eta_{\mu \lambda} s_{, \nu}^{\lambda}+\eta_{\nu \lambda} s_{, \mu}^{\lambda}+\eta_{\lambda \tau} s_{, \mu}^{\lambda} s_{, \nu}^{\tau}\right) \frac{d x^{\mu}}{d \tau} \frac{d x^{\nu}}{d \tau} d \tau .
\end{aligned}
$$

Second, we assume that $I_{q}$ originates from a change of the flat metric $\eta_{\mu \nu}$ to another metric $g_{\mu \nu}$ with the path still given by $x^{\mu}(\tau)$, that is,

$$
I_{q}=\int_{\tau_{A}}^{\tau_{B}} g_{\mu \nu}(x) \frac{d x^{\mu}}{d \tau} \frac{d x^{\nu}}{d \tau} d \tau .
$$

These two forms being equivalent representations for $I_{q}$, they must give the same value, therefore we must have

$$
g_{\mu \nu}(x)=\eta_{\mu \nu}+\eta_{\mu \lambda} s_{, \nu}^{\lambda}+\eta_{\nu \lambda} s_{, \mu}^{\lambda}+\eta_{\lambda \tau} s_{, \mu}^{\lambda} s_{, \nu}^{\tau} .
$$

Here, when we identify (51) and (52) as two equivalent forms for $I_{q}$ we are implicitly assuming that the net effect of the fluctuations of the spacetime coordinates $x \rightarrow x+s$ is to change the flat metric to an effective metric $g_{\mu \nu}$.

\section{Conclusion}

We investigated a particular type of nonlinear transformation and the corresponding invariant interval $I_{q}=x^{2}+\hat{f}$ $\left(\hat{f}=s^{2} f^{2}\right)$. This interval was conceived as a change of the standard spacetime interval $I=x^{2}$. One natural question that arises is on the physical principles that determine a change like $I \rightarrow I_{q}$. The original argument proposed by Dresden $[4,5]$ that we revisited in Section 4.1 gives just a partial answer since it is excessively qualitative and does not contemplate causes other than the uncertainty principle in the position. In our model, we build $\hat{f}$ from sections $s: U \subseteq M \rightarrow T M$, then to answer that question we must first give a physical interpretation for the sections. Here, the assumption of extra structure in spacetime is not new [13-15], for example, in his model for the ether, Dirac [13] assumed that in spacetime there is defined a velocity distribution $v^{\mu}$ that is associated to the gauge field $A^{\mu}$, for example, $k^{-1} A^{\mu}=v^{\mu}$. In another direction, Caianiello et al. 
[15] assumed there is defined a field of velocities in spacetime corresponding to the movement of test particles of mass $m$. This, of course, introduces an unnecessary element in spacetime (the test particles) and the problem is still open if we intend to rely only on elements that are intrinsic to the spacetime.

The nonlinear transformation also raises a question about causality. In fact, Zeeman showed that causality implies the usual (linear) Lorentz transformations [16]. Then, we would expect any model that incorporates nonlinear transformations violates causality. However, this is not necessarily true for the nonlinear transformations because Zeeman's result is verified for the spacetime $M$, not for the extended space $M \oplus \Gamma(T M)$ where the nonlinear transformations were defined, therefore, Zeeman's argument may need some modification or not even apply.

Our investigation may be extended to the case spacetime is a manifold. Now, the relevant spacetime structure becomes the fundamental metric form $I:=\eta_{\mu \nu} d x^{\mu} d x^{\nu}$, and we are interested in changes of $I=\eta_{\mu \nu} d x^{\mu} d x^{\nu} \rightarrow I_{q}:=\eta_{\mu \nu} d x^{\mu} d x^{\nu}+$ $\eta_{\mu \nu} d s^{\mu} d s^{\nu}$. Here, the problem is to find the analogue of $M \oplus$ $\Gamma(T M)$ and the corresponding transformations that leave $I_{q}$ invariant.

Nowadays, nonlinear transformations are being studied intensively in the context of the Double Special Relativity (DSR) models, where the nonlinear transformation is defined in the momenta space. A related problem in these models is to find the corresponding coordinate transformation. In [3], it was shown that the Fock transformation [1] was the counterpart in the coordinates of a certain transformation in the momenta. Then, in a similar way, it may be worth to investigate if the Albano-Dresden transformation corresponds to a nonlinear transformation in the momenta space of some DSR model.

In our work, the group of nonlinear transformations of the Albano-Dresden type was realized in an infinite dimensional space $M \oplus \prod_{x \in M} E_{x}$. The infinite dimensionality of the realization stands for the space $\prod_{x \in M} E_{x}$ where the sections were defined. We notice that Albano and Dresden obtained heuristically their transformation in a finite 4dimensional spacetime $M$ with no recourse to sections. However, as we have shown at the end of Section 2, their transformation is a particular case of type I transformations when we take sections $s$ satisfying $s^{2}=1$. Due to the heuristic approach followed by Albano and Dresden, it is not clear the details behind the process they employed to construct their realization. Therefore, it is not clear if other nonlinear realization of the Lorentz transformations may be made in a finite dimensional space, or even if other infinite dimensional realizations are related to the same infinite dimensional space we have employed.

Finally, it is natural to interpret the function $f$ present in the Albano-Dresden interval $I_{q}=x^{2}+s^{2} f^{2}$ as the gauge function of a Lyra manifold. In fact, for a Lyra manifold the gauge function is intrinsically defined in the manifold as part of the local chart $(U, \varphi)$ that is then written as $(U, \varphi, f)$ [9]. Charts like this were also of fundamental importance in the interpretation of the nonlinear transformation and its linearization in terms of compatible atlas as given in
Section 3. For a Weyl manifold, the analogue of the gauge function becomes a map $\lambda: M \rightarrow R$ that appears related to the gauge transformation of the metric $[17,18]$. Then, the role of the gauge function in Weyl and Lyra geometries are quite different. It seems there is no impossibility to define in the Weyl manifold intrinsic functions $f_{i}: U_{i} \rightarrow R$, or even to use $\lambda$ to introduce a new basis for $T_{p} M$, repeating the same construction that was made in Section 3 for the Lyra manifold. However, this would extend considerably the definition of the Weyl manifold introducing by hand extra structure that is not typical of this kind of space.

\section{Acknowledgments}

The authors thank all the staff of the library of Centro Brasileiro de Pesquisas Físicas (CBPF). M. Carvalho thanks Aurelina Carvalho, Carlinda Ferreira Lima, José E. Carvalho, Aureliana Cabral Raposo. A. Lyra thanks Maria I. Lyra and Eliana N. Lyra de Oliveira for the permanent support. ICXC Nika.

\section{References}

[1] V. A. Fock, The Theory of Space, Time and Gravitation, Macmillan, New York, NY, USA, 1964.

[2] J. Gauthier and L. Marchildon, "Constraints on nonlinear extensions of the Lorentz group," Physical Review D, vol. 35, no. 10, pp. 3060-3065, 1987.

[3] D. Kimberly, J. Magueijo, and J. Medeiros, "Nonlinear relativity in position space," Physical Review D, vol. 70, no. 8, Article ID 084007, 2004.

[4] M. Dresden, "The universality of Lorentz invariancein particle physics," in Proceedingsof Conference on Non-Compact Groups in Particle Physics, Y. Chow, Ed., pp. 103-118, Benjamin, New York, NY, USA, 1966.

[5] M. Dresden, "The existence and significance of nonlinear realization of the Poincaré group," in Proceedings of the Coral Gables Conference on Fundamental Interactionsat High Energy II, A. Perlmutter, G. Iverson, and R. Williams, Eds., pp. 279289, Gordon and Breach, New York, NY, USA, 1970.

[6] A. Albano and M. Dresden, "Nonlinear spacetime transformations related to the Lorentz group," Proceedings of the National Academy of Sciences of the United States of America, vol. 58, pp. 916-922, 1967.

[7] M. Dresden and A. Albano, "A class of nonlinear realization of the Poincaré group," Journal of Mathematical Physics, vol. 13, pp. 275-282, 1972.

[8] L. Chambadal and J. L. Ovaert, Algèbre Linéaire et Algèbre Tensorielle, Dunod, Paris, France, 1968.

[9] D. K. Sen and K. A. Dunn, "A scalar-tensor theory of gravitation in a modified Riemannian manifold," Journal of Mathematical Physics, vol. 12, no. 4, pp. 578-586, 1971.

[10] T. Asselmeyer-Maluga and C. H. Brans, Exotic Smoothness and Physics: Differential Topology and Spacetime Models, World Scientific, Singapore, 2007.

[11] I. Robinson and Y. Neeman, "Seminar on the embedding problem," Reviews of Modern Physics, vol. 37, p. 201, 1965.

[12] R. E. Greene, "Isometric embeddings of Riemannian and pseudo Riemannian manifolds," Memoirs of the American Mathematical Society, vol. 97, pp. 1-63, 1970. 
[13] P. A. M. Dirac, "Is there an ether?" Nature, vol. 24, pp. 906907, 1951.

[14] M. Carvalho and A. L. Oliveira, "A new version of the Dirac's ether and its cosmological applications," Foundations of Physics Letters, vol. 16, pp. 255-263, 2003.

[15] E. R. Caianiello, A. Feoli, M. Gasperini, and G. Scarpetta, "Quantum corrections to the spacetime metric from geometric phase space quantization," International Journal of Theoretical Physics, vol. 29, no. 2, pp. 131-139, 1990.

[16] E. C. Zeeman, "Causality implies the Lorentz group," Journal of Mathematical Physics, vol. 5, no. 4, pp. 490-493, 1964.

[17] D. K. Sen and J. R. Vanstone, "On Weyl and Lyra manifolds," Journal of Mathematical Physics, vol. 13, no. 7, pp. 990-993, 1972.

[18] G. Folland, "Weyl manifolds," Journal of Differential Geometry, vol. 4, pp. 145-153, 1970. 

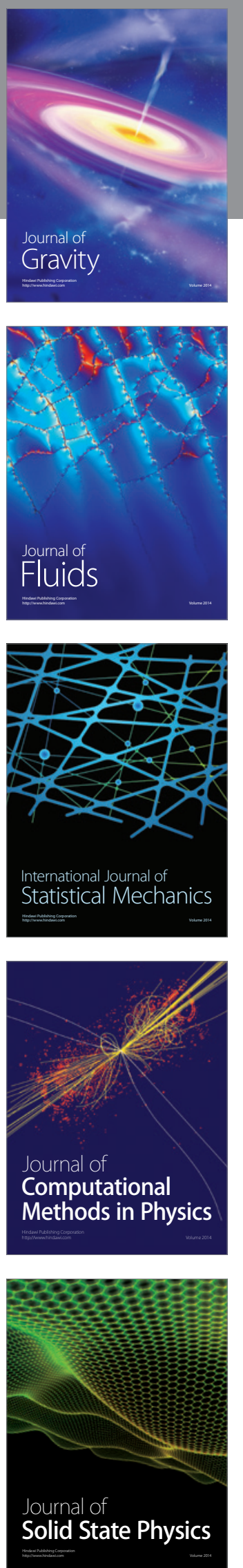

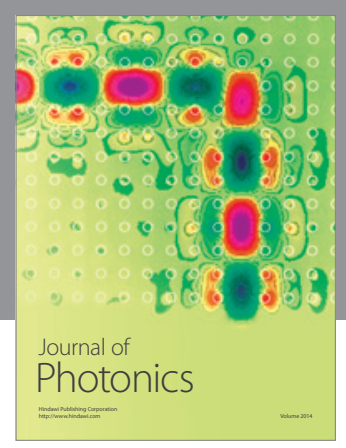

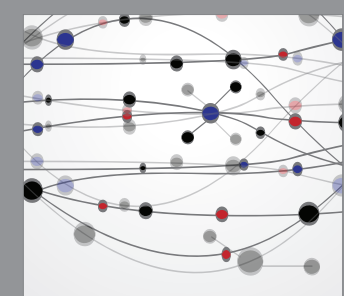

The Scientific World Journal
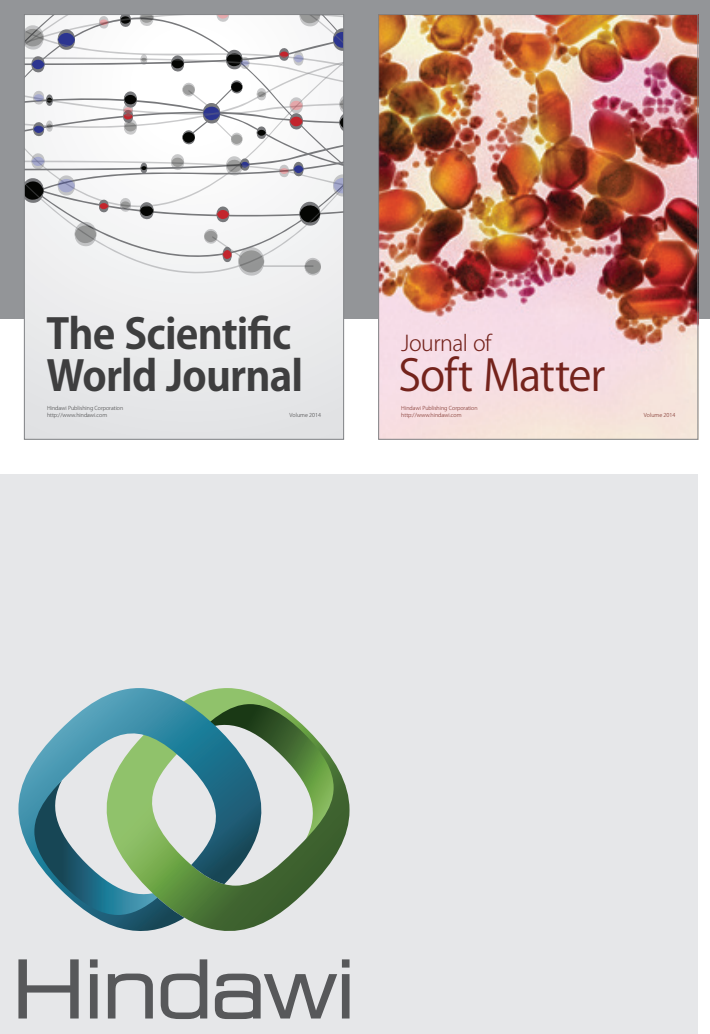

Submit your manuscripts at

http://www.hindawi.com
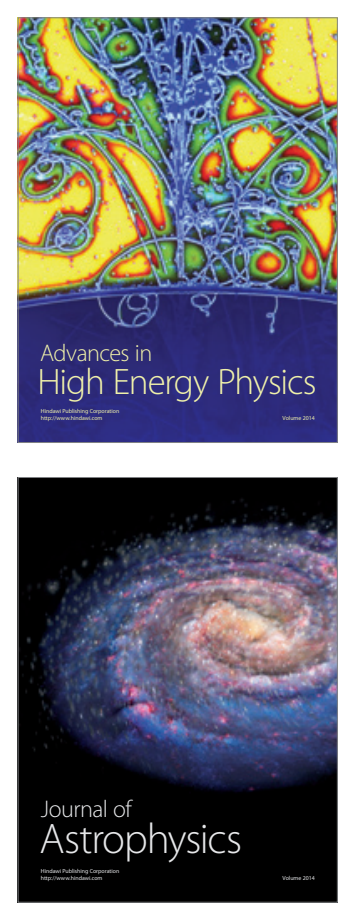
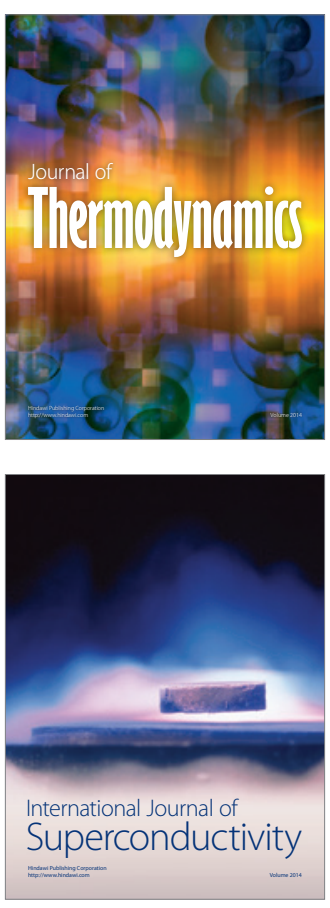
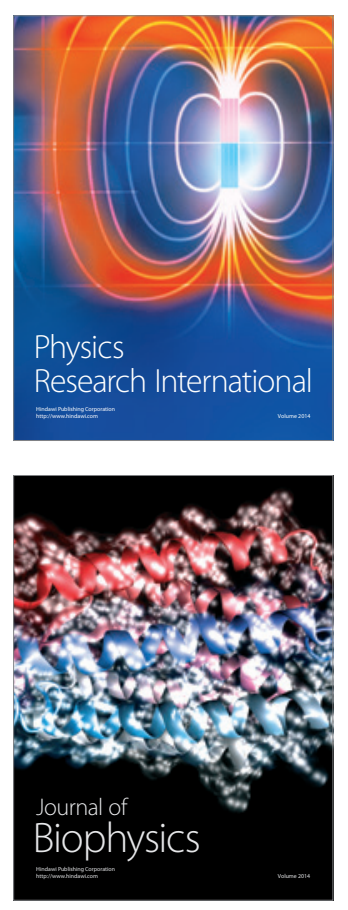
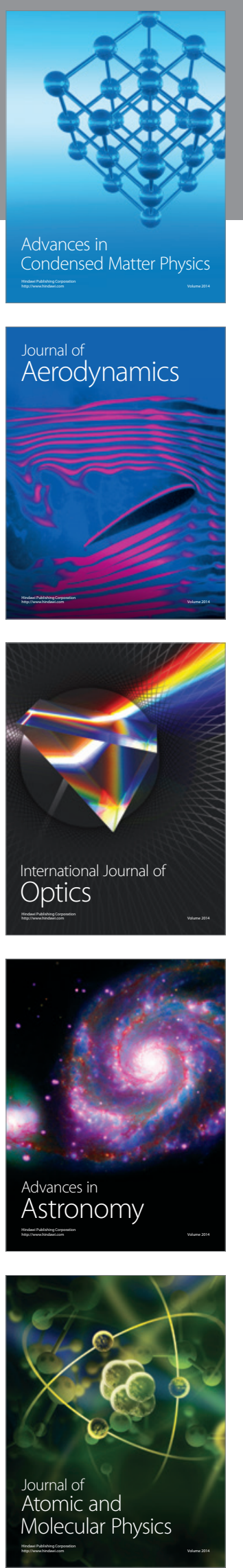